\title{
Lung function in patients with primary ciliary dyskinesia: an iPCD Cohort study
}

\author{
Florian S. Halbeisen ${ }^{1}$, Myrofora Goutaki $\mathbb{1}^{1,2}$, Ben D. Spycher ${ }^{1,2}$, \\ Israel Amirav $\mathbb{1}^{3,4,5}$, Laura Behan ${ }^{6,7}$, Mieke Boon $^{8}$, Claire Hogg 9 , \\ Carmen Casaulta ${ }^{2,10}$, Suzanne Crowley ${ }^{11}$, Eric G. Haarman ${ }^{12}$, Bulent Karadag ${ }^{13}$, \\ Cordula Koerner-Rettberg ${ }^{14}$, Michael R. Loebinger ${ }^{15}$, Henryk Mazurek ${ }^{16}$, \\ Lucy Morgan ${ }^{17}$, Kim G. Nielsen ${ }^{18}$, Heymut Omran ${ }^{19}$, Francesca Santamaria ${ }^{20}$, \\ Nicolaus Schwerk ${ }^{21}$, Guillaume Thouvenin ${ }^{22,23,24}$, Panayiotis Yiallouros ${ }^{25}$, \\ Jane S. Lucas (106 ${ }^{6}$, Philipp Latzin (10) ${ }^{2}$ and Claudia E. Kuehni (10 ${ }^{1,2}$
}

@ERSpublications

PCD affects lung function from an early age, similarly to CF, thus early multidisciplinary management is crucial http://ow.ly/T9ch30kHXns

Cite this article as: Halbeisen FS, Goutaki M, Spycher BD, et al. Lung function in patients with primary ciliary dyskinesia: an iPCD Cohort study. Eur Respir J 2018; 52: 1801040 [https://doi.org/10.1183/ 13993003.01040-2018].

ABSTRACT Primary ciliary dyskinesia (PCD) has been considered a relatively mild disease, especially compared to cystic fibrosis (CF), but studies on lung function in PCD patients have been few and small.

This study compared lung function from spirometry of PCD patients to normal reference values and to published data from CF patients. We calculated $\mathrm{z}$-scores and \% predicted values for forced expiratory volume in $1 \mathrm{~s}$ (FEV1) and forced vital capacity (FVC) using the Global Lung Function Initiative 2012 values for 991 patients from the international PCD Cohort. We then assessed associations with age, sex, country, diagnostic certainty, organ laterality, body mass index and age at diagnosis in linear regression models. Lung function in PCD patients was reduced compared to reference values in both sexes and all age groups. Children aged 6-9 years had the smallest impairment (FEV1 z-score -0.84 ( -1.03 to -0.65 ), FVC z-score -0.31 ( -0.51 to -0.11$)$ ). Compared to CF patients, FEV1 was similarly reduced in children (age 6-9 years PCD 91\% (88-93\%); CF 90\% (88-91\%)), but less impaired in young adults (age 1821 years PCD 79\% (76-82\%); CF 66\% (65-68\%)). The results suggest that PCD affects lung function from early in life, which emphasises the importance of early standardised care for all patients. 
Affiliations: ${ }^{1}$ Institute of Social and Preventive Medicine, University of Bern, Bern, Switzerland. ${ }^{2}$ Paediatric Respiratory Medicine, Children's University Hospital of Bern, University of Bern, Bern, Switzerland. ${ }^{3}$ The PCD Israeli Consortium. ${ }^{4}$ Dept of Pediatrics, Faculty of Medicine, Bar Ilan University, Ramat Gan, Israel. ${ }^{5}$ Dept of Pediatrics, University of Medicine, Edmonton, AB, Canada. ${ }^{6}$ Primary Ciliary Dyskinesia Centre, NIHR Respiratory Biomedical Research Centre, University of Southampton, Southampton, UK. ${ }^{7}$ School of Applied Psychology, University College Cork, Cork, Ireland. ${ }^{8}$ Dept of Paediatrics, University Hospital Gasthuisberg, Leuven, Belgium. ${ }^{9}$ Dept of Paediatrics, Primary Ciliary Dyskinesia Centre, Royal Brompton and Harefield Foundation Trust, London, UK. ${ }^{10}$ The Swiss PCD Group. ${ }^{11}$ Unit for Paediatric Heart, Lung, Allergic Diseases, Rikshospitalet, Oslo, Norway. ${ }^{12}$ Dept of Pediatric Pulmonology, VU University Medical Center, Amsterdam, The Netherlands. ${ }^{13}$ Dept of Pediatric Pulmonology, Marmara University, School of Medicine, Istanbul, Turkey. ${ }^{14}$ Dept of Paediatric Pneumology, University Children's Hospital of Ruhr University Bochum, Bochum, Germany. ${ }^{15}$ Host Defence Unit, Royal Brompton and Harefield NHS Foundation Trust, London, UK. ${ }^{16}$ Dept of Pneumonology and Cystic Fibrosis, Institute of Tuberculosis and Lung Disorders, Rabka-Zdrój, Poland. ${ }^{17}$ Dept of Respiratory Medicine, Concord Hospital Clinical School, University of Sydney, Sydney, Australia. ${ }^{18}$ Danish PCD Centre Copenhagen, Paediatric Pulmonary Service, Copenhagen University Hospital, Copenhagen, Denmark. ${ }^{19}$ Dept of General Paediatrics and Adolescent Medicine, University Hospital Muenster, Muenster, Germany. ${ }^{20}$ Dept of Translational Medical Sciences, Federico II University, Napoli, Italy. ${ }^{21}$ Clinic for Paediatric Pulmonology, Allergiology and Neonatology, Hannover Medical School, Hannover, Germany. ${ }^{22}$ The French Reference Centre for Rare Lung Diseases. ${ }^{23}$ Paediatric Pulmonary Dept, Trousseau Hospital APHP, Sorbonne Universities and Pierre et Marie Curie University, Paris, France. ${ }^{24}$ INSERM U938-CRSA, Paris, France.

${ }^{25}$ Medical School, University of Cyprus, Nicosia, Cyprus.

Correspondence: Claudia E. Kuehni, Institute of Social and Preventive Medicine, University of Bern, Finkenhubelweg 11, 3012 Bern, Switzerland.

E-mail: claudia.kuehnidispm.unibe.ch

\section{Introduction}

Primary ciliary dyskinesia (PCD) is a multiorgan disease which is genetically and clinically heterogeneous $[1,2]$. PCD affects $\sim 1: 10000$ people, and it is often underdiagnosed or diagnosed late $[3,4]$. Due to abnormal ciliary function in PCD, mucus is not cleared properly from the airways [5], resulting in chronic lung disease. For many years, PCD was considered a relatively mild disease [6], particularly when compared to cystic fibrosis (CF).

Findings on lung function of PCD patients in prior studies are inconsistent. Children with PCD from six North American centres were found to have normal spirometric indices, with a median forced expiratory volume in $1 \mathrm{~s}\left(\mathrm{FEV}_{1}\right)$ of $89 \%$ pred [7]. In contrast, a Belgian study reported that 112 patients with PCD already had abnormal FEV1 and forced vital capacity (FVC) from the age of 5 years [8]. Recent studies, particularly in adults, have reported considerable heterogeneity among patients [9-11]. A recent systematic review of 24 studies [12] found that FEV1 of patients with PCD ranged from 44\% pred to 69\% pred. Most studies were small, with an average of 40 patients per study. While risk factors for lung function impairment have been studied in many respiratory diseases, this has not been done for PCD.

Using data from the international PCD (iPCD) Cohort [13], we assessed lung function in patients with PCD and compared it to Global Lung Function Initiative (GLI) 2012 reference values [14], and to published lung function data of patients with CF [15]. We investigated possible determinants of lung function including sex, age, situs anomalies, body mass index (BMI), country of residence, ultrastructural defect and level of diagnostic certainty. In addition, we assessed whether age at diagnosis in paediatric patients is associated with lung function later in life.

\section{Methods}

\section{Study population and study design}

The iPCD Cohort is a large, retrospective international cohort initiated during the European Union FP7 project Better Experimental Screening and Treatment for Primary Ciliary Dyskinesia (BESTCILIA) and further developed through COST-Action network BEAT-PCD [16]. It includes international information on diagnostic test results, clinical symptoms, growth, lung function and treatment for $>3200$ patients [13]. For this study, we included all patients whose datasets had been delivered, cleaned and standardised by the time of analysis (April 2016), and for whom we had information on FEV1 and FVC. Participating centres were requested to only deliver lung function data of clinical stable patients from scheduled follow-up visits. Principal investigators were responsible for obtaining ethics approval and informed consent in their countries to contribute observational, anonymised data.

\section{PCD diagnosis}

PCD diagnostics remains challenging and has evolved over the years [17]. Recent guidelines recommend a combination of tests [18], but test availability differs between countries [19]. In the iPCD Cohort, we divided patients into three subgroups based on diagnostic tests. The first subgroup includes patients with 
definite PCD defined according to recent guidelines of the European Respiratory Society PCD diagnostics task force [18], as patients with a clinical phenotype consistent with PCD and a hallmark transmission electron microscopy (TEM) finding, and/or pathogenic biallelic PCD genetic mutations. The second subgroup, probable PCD, includes patients with a clinical phenotype consistent with PCD and abnormal high-speed video microscopy findings and/or low nasal nitric oxide and a clinical phenotype consistent with PCD. The third subgroup includes patients with clinical phenotypes consistent with PCD diagnosis in whom other possible diagnoses such as CF were excluded. In most of these patients the diagnostic algorithm was not complete due to test unavailability (e.g. genetic testing was not available for most centres). The online supplementary material describes details of diagnosis.

\section{Lung function measurements}

We calculated FEV1 and FVC z-scores adjusted for age, sex, height and ethnicity, and \% pred values using the GLI 2012 reference values [14]. We excluded data from children who were aged $<6$ years to ensure better measurement quality and comparability with the published CF data [15]. We included one lung function measurement per patient in the analysis. For patients with multiple measurements, we used the measurement recorded at the youngest age.

\section{Determinants of lung function}

We investigated the association of lung function with sex, age, country of residence, level of diagnostic certainty, organ laterality, BMI and ultrastructural defect at time of lung function measurement. Details on how we categorised the selected variables and why we chose them are available in the online supplementary material.

\section{Comparison to CF patients}

For comparison with CF patients, we used recently published data of 5904 patients with CF from the UK [15], which described \% pred values for FEV1 and FVC, instead of z-scores. Thus, we analysed our PCD data in the same way to allow for a direct comparison. For this analysis, we stratified the data by age without further adjustment.

\section{Statistical analysis}

We compared included patients with those who were excluded due to the absence of lung function measurements using Chi-squared tests. We compared FEV1 and FVC to the GLI reference values, and investigated potential determinants of lung function including sex, age, country and diagnostic certainty using multivariable linear regression models with $\mathrm{z}$-scores as dependent variable. We tested for differences in FEV1 and FVC between patient groups by performing likelihood ratio tests. We coded categorical predictor variables included in regression models using weighted effect coding. Estimated parameters for a given category thus represent mean $z$-score differences from the population mean $[20,21]$. In separate regression models including patients with available data and adjusting for the same variables, we assessed the association between BMI, organ laterality and ultrastructural defect with FEV1 and FVC. In a separate model applied to our paediatric population, we assessed whether age at diagnosis influenced lung function later in life. We received date of diagnosis from the centres or calculated it from the dates of diagnostic test results. In patients without positive diagnostic test results, we defined date of clinical diagnosis as the date of the first clinical follow-up. For this analysis, we only included children who had lung function measurements available after diagnosis $(n=167)$ to ensure that they were already under PCD management at lung function measurement. We included all patients diagnosed with PCD between birth and the age of 15 years who had at least one available lung function measurement between the ages 15 and 20 years (online supplementary figure S2). We categorised age at diagnosis in three groups: $0-5$ years ( $\mathrm{n}=36$ ), $5-10$ years $(n=65)$ and $10-15$ years $(n=65)$. We performed two sensitivity analyses to test the robustness of findings, the first by including only patients with definite PCD diagnosis [18], and the second by including the latest recorded lung function measurement for patients with multiple recorded measurements, instead of the earliest. We used STATA 14.1 (StataCorp, College Station, TX, USA) and R 3.1.2 (www.r-project.org) for all analyses.

\section{Results}

Population characteristics

21 centres had delivered cleaned and standardised data from 2667 patients by the time of analysis (April 2016). After exclusion of patients aged $<6$ years and those with no lung function data or information insufficient to calculate z-scores, we included 991 patients in the main analysis (online supplementary figure S1). Characteristics of the main study population are given in table 1, while online supplementary table S2 compares included and excluded patients. 
In total, $62 \%$ of included patients were designated as definite PCD, $21 \%$ as probable PCD and $17 \%$ as having only a clinical diagnosis (table 1). Most patients had been diagnosed during childhood. Included patients differed from those excluded because of insufficient data to calculate lung function z-scores by age and country of residence, and due to the lack of lung function data by sex and country of residence (online supplementary table S2).

\section{FEV1 and FVC in patients with PCD}

Mean FEV1 and mean FVC were lower than the mean reference values in all age groups. Among all patients, $46 \%$ had an FEV1 z-score below the lower limit of normal (LLN) of -1.645 and $28 \%$ had an FVC $\mathrm{z}$-score <LLN. Impairment differed between age groups (table 2, figure 1). Children aged 6-9 years had the best lung function and adults the worst. Results differed between countries. PCD patients had lower FEV1 Z-scores than reference data in all countries and lower FVC z-scores in most countries (table 2, figure 2), but patients in Belgium, France, Italy and the Netherlands had on average better FVC z-scores than rest of the study population and were comparable to the GLI reference values. Female patients had lower FEV 1 z-scores than male patients (table 2, figure 3), but their FVC was comparable. FEV1 and FVC z-scores did not differ by levels of diagnostic certainty, and there was no evidence of association with organ laterality (figure 3 and online supplementary table S3). BMI was positively associated with FEV1 and FVC: patients

\begin{tabular}{|c|c|}
\hline \multicolumn{2}{|l|}{ Sex } \\
\hline Male & 487 (49) \\
\hline Female & $504(51)$ \\
\hline \multicolumn{2}{|l|}{ Country of residence ${ }^{\#}$} \\
\hline Australia & $34(3)$ \\
\hline Northern Europe & $306(31)$ \\
\hline Western Europe & $392(40)$ \\
\hline Eastern Europe & 74 (8) \\
\hline Southern Europe & $42(4)$ \\
\hline Western Asia & 143 (14) \\
\hline \multicolumn{2}{|l|}{ Organ laterality } \\
\hline Situs solitus & $499(51)$ \\
\hline Situs inversus & 348 (35) \\
\hline Heterotaxia & $13(1)$ \\
\hline Situs status not reported & $131(13)$ \\
\hline \multicolumn{2}{|l|}{ Time period of birth } \\
\hline Before 1976 & 151 (15) \\
\hline 1977-1996 & 363 (37) \\
\hline $1997-2015$ & 477 (48) \\
\hline \multicolumn{2}{|l|}{ Diagnostic information } \\
\hline Definite PCD diagnosis" & $611(62)$ \\
\hline Probable PCD diagnosis $^{+}$ & $207(21)$ \\
\hline Clinical diagnosis only & 173 (17) \\
\hline \multicolumn{2}{|l|}{ Age at diagnosis } \\
\hline $0-9$ years & 448 (45) \\
\hline $10-19$ years & 317 (32) \\
\hline $20-29$ years & 98 (10) \\
\hline $30-39$ years & $61(6)$ \\
\hline 40-49 years & $36(4)$ \\
\hline$\geqslant 50$ years & $31(3)$ \\
\hline \multicolumn{2}{|l|}{$\mathrm{BMI} \mathrm{S}^{\S}$} \\
\hline Underweight & $59(6)$ \\
\hline Normal & $752(81)$ \\
\hline Overweight & 116 (13) \\
\hline
\end{tabular}

Data are presented as $\mathrm{n}(\%) . \mathrm{n}=991$. PCD: primary ciliary dyskinesia; BMI: body mass index. \#: based on geographical region definitions of the United Nations Statistics Division (March 2017). Northern Europe: Denmark, Norway, UK; Western Europe: Belgium, France, Germany, Switzerland, the Netherlands; Eastern Europe: Poland; Southern Europe: Italy, Serbia; Western Asia: Cyprus, Israel, Turkey. ๆ: defined as hallmark PCD electron microscopy findings and/or biallelic gene mutation identified based on the European Respiratory Society PCD diagnostics guidelines [18]. + : abnormal light or high-frequency video microscopy finding and/or low $\left(\leqslant 77 \mathrm{~nL} \cdot \mathrm{min}^{-1}\right)$ nasal nitric oxide value. §: BMI $z$-scores $\leqslant-1.96$ underweight, $-1.96-<1.96$ normal, $\geqslant 1.96$ overweight. 
TABLE 2 Forced expiratory volume in $1 \mathrm{~s}$ (FEV1) and forced vital capacity (FVC) in patients with primary ciliary dyskinesia (PCD) from the international PCD Cohort, compared to Global Lung Function Initiative 2012 reference values

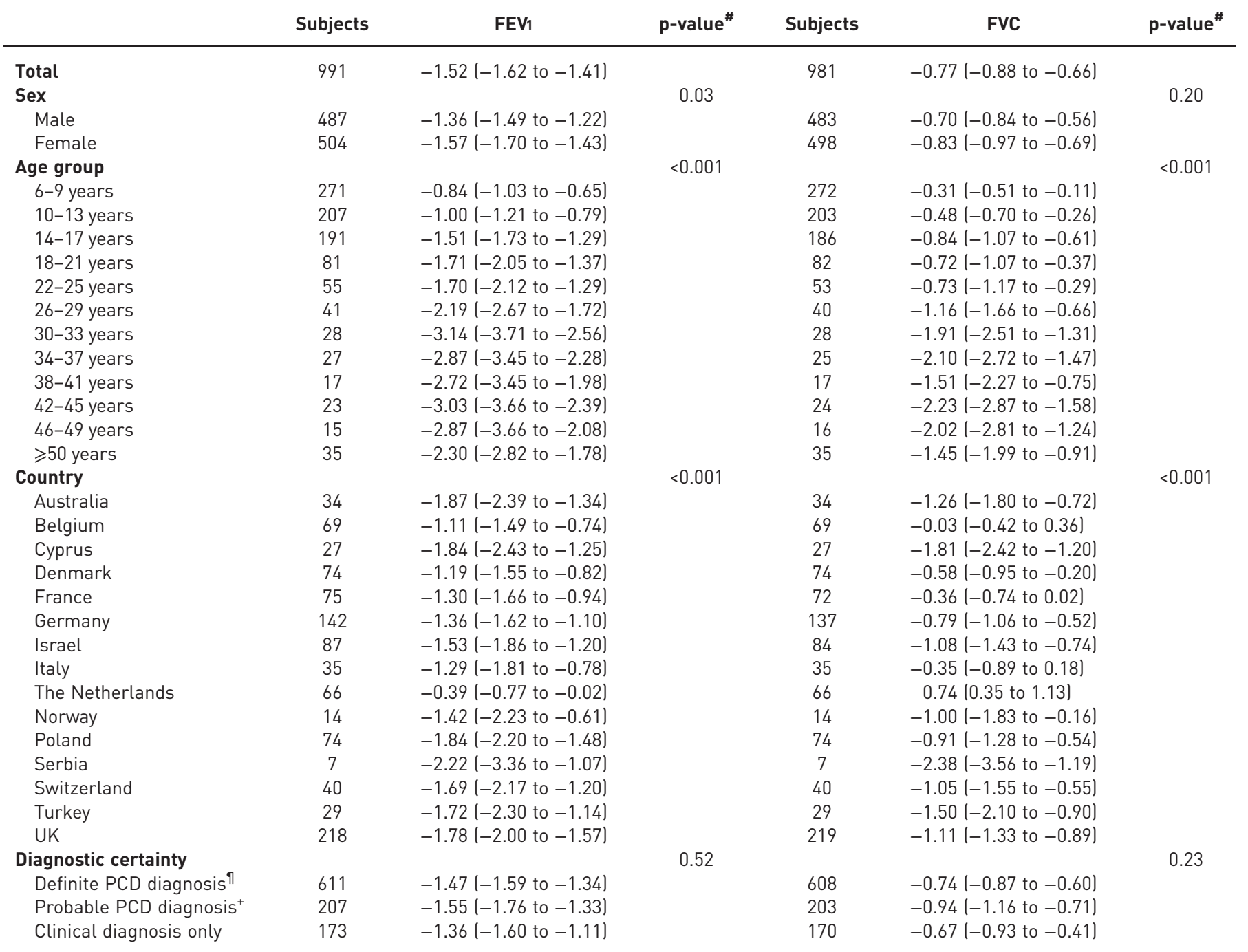

Data are presented as $\mathrm{n}$ or mean z-scores $(95 \% \mathrm{Cl})$ for each group after adjusting for the remaining characteristics, unless otherwise stated. $\mathrm{n}=991 .{ }^{\text {\# }}$ : likelihood ratio test $\mathrm{p}$-value indicating whether the characteristic explains differences in FEV1 or FVC within the study population; ף: defined as hallmark PCD electron microscopy findings and/or biallelic gene mutation identified based on the European Respiratory Society PCD diagnostics guidelines [18]; ${ }^{+}$: abnormal light or high-frequency video microscopy finding and/or low nasal nitric oxide value.

who were underweight had worse lung function than normal and overweight patients (figure 3 and online supplementary table S4). FEV1 z-scores differed between ultrastructural defect groups; patients with a microtubular defect had worse lung function than patients with a non-diagnostic TEM and patients with outer or inner dynein arm defects (table 3). Sensitivity analyses that included only patients with a definite PCD diagnosis $(n=611)$ had similar results for FEV1 and FVC to the original models (table 2 and online supplementary table S5). When we included the latest recorded lung function measurement for patients with multiple available values instead of the earliest available, results were similar to the original models (table 2 and online supplementary table S6).

Age at diagnosis was not significantly associated with lung function in 167 patients aged 15-20 years. Among them, the children diagnosed at ages 5-10 years had better lung function compared to those diagnosed at 10-15 years; however, differences were small and not statistically significant (online supplementary figure S3).

Lung function in patients with PCD compared to patients with CF

Both PCD and CF patients had impaired spirometric indices (figure 4). Average FEV1 and FVC values of children with PCD who were aged $<17$ years were low and similar to children with CF. During 

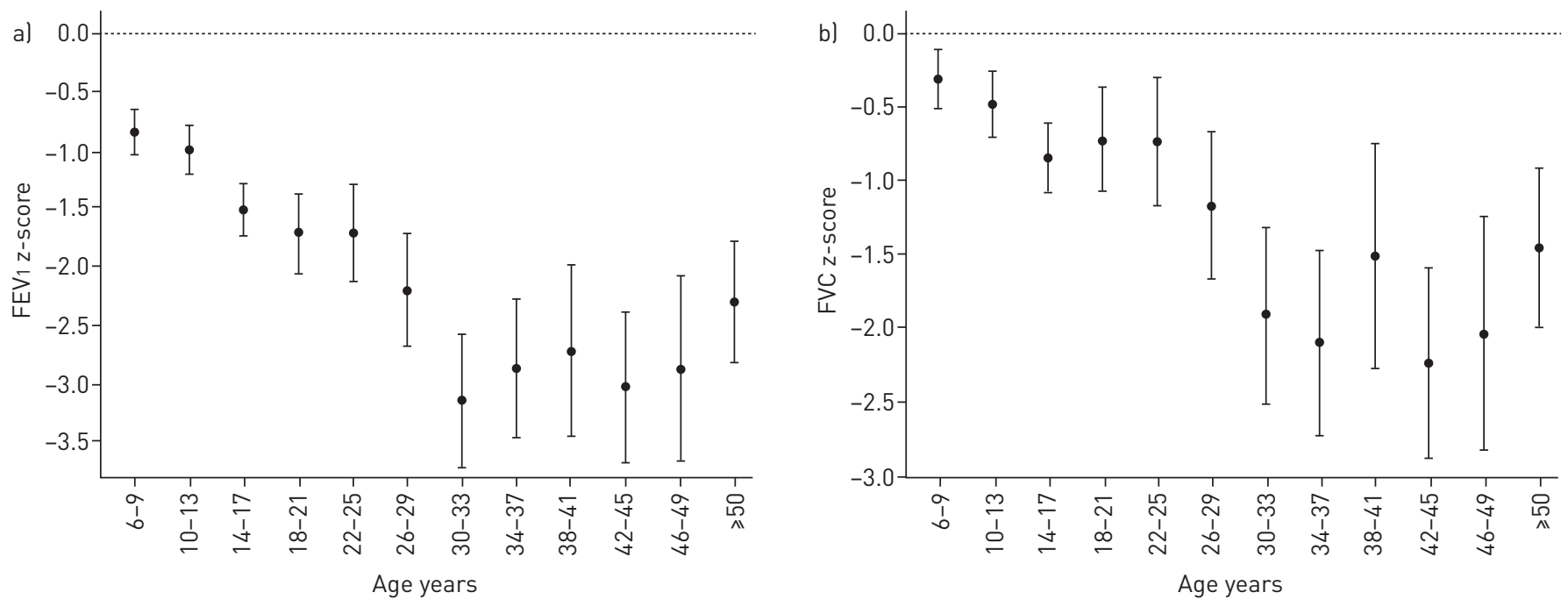

FIGURE 1 a) Forced expiratory volume in $1 \mathrm{~s}(\mathrm{FEV} 1)$ and b) forced vital capacity (FVC) in primary ciliary dyskinesia patients by age group compared to Global Lung Function Initiative 2012 reference values. FEV1 and FVC are presented as mean z-score (95\% CI) after adjusting for sex, country and level of diagnostic certainty. The dashed line shows the mean z-score of the normal population.

adolescence and early adulthood, measurements began to diverge and older patients with PCD had better lung function than CF patients. Although differences in FEV1 and FVC persisted between PCD and CF patients, after the age of 30 years the differences narrowed.

\section{Discussion}

This multinational study found that lung function of patients with PCD was considerably lower than reference values. All age groups, including young children and patients from most countries had reduced FEV1 and FVC z-scores. Female sex, underweight and type of ultrastructural defect were predictors of worse lung function. Results were consistent in all diagnostic and organ laterality subgroups. In children and adolescents, lung function of patients with PCD was similar to that of CF patients. Our data therefore refute the traditionally held assumption that PCD is a relatively mild disease.
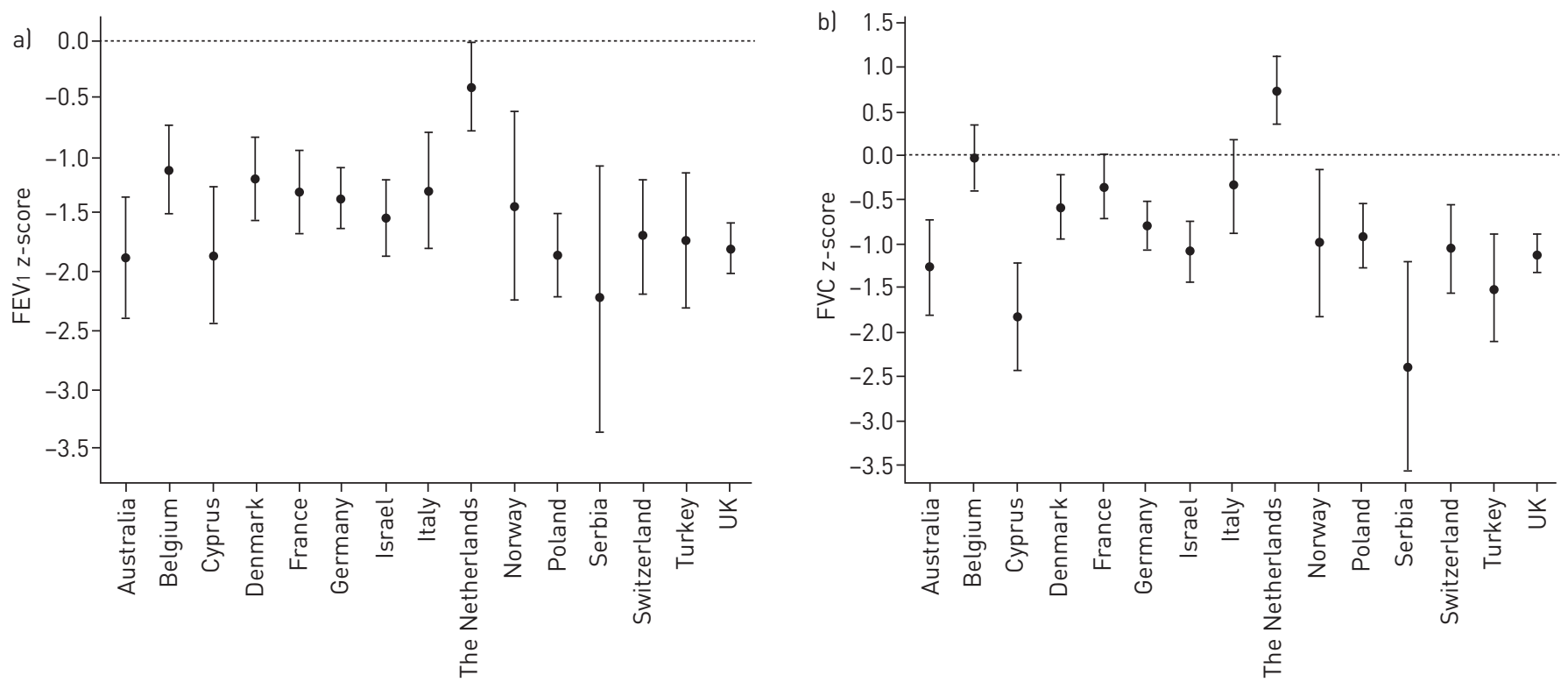

FIGURE 2 a) Forced expiratory volume in $1 \mathrm{~s}(\mathrm{FEV} 1)$ and b) forced vital capacity (FVC) in primary ciliary dyskinesia patients by country of residence compared to Global Lung Function Initiative 2012 reference values. FEV1 and FVC are presented as mean z-score (95\% Cl) after adjusting for sex, age group and level of diagnostic certainty. The dashed line shows the mean z-score of the normal population. 

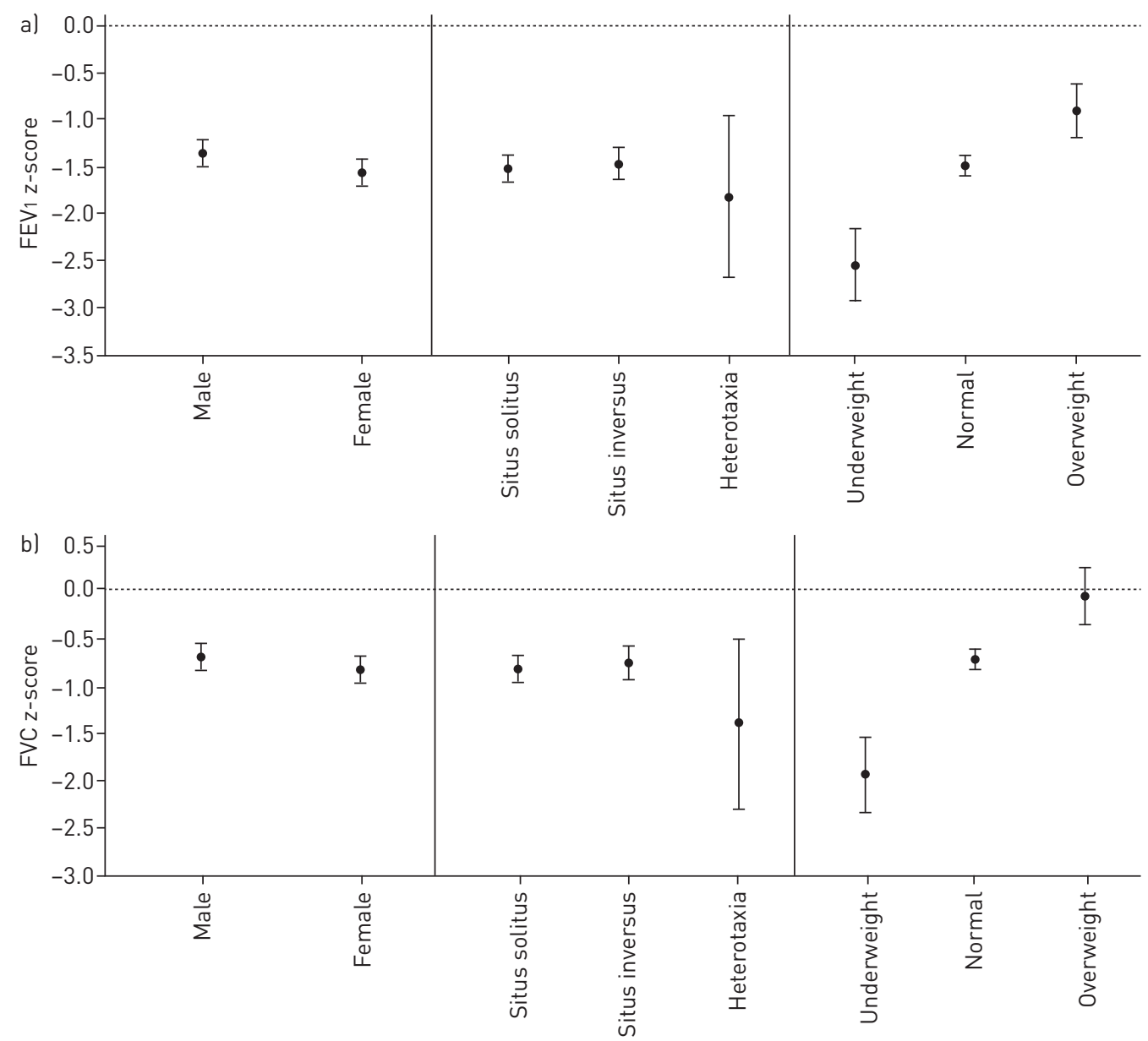

FIGURE 3 a) Forced expiratory volume in $1 \mathrm{~s}$ (FEV 1 ) and b) forced vital capacity (FVC) in primary ciliary dyskinesia patients by sex, situs anomalies and body mass index compared to Global Lung Function Initiative 2012 reference values. FEV 1 and FVC are presented as mean z-score $(95 \% \mathrm{CI})$ after adjusting for age, country and level of diagnostic certainty. The dashed line shows the mean z-score of the normal population.

This is the largest multicentre study on lung function in patients with PCD. The large study population enabled us to study differences in FEV1 and FVC between males and females, age groups, countries and levels of diagnostic certainty. A potential limitation of our study is that the iPCD Cohort includes mainly patients from centres with developed diagnostic facilities. Therefore, smaller centres and some countries are under-represented. In addition, we had no data on infection status at time of measurement, such as chronic colonisation with Pseudomonas. In our analysis we only included stable patients measured at scheduled follow-up visits. Only a prospective study could investigate this association, because surveillance of infection varies between countries and centres. Centres that routinely assess microbiology are more likely to detect pathogens than centres that check patients when they are unwell. A limitation of the comparison with CF patients was that CF patients came from the UK only, and their lung function was compared using Wang-Hankison reference values, while GLI reference values were used for the PCD patients. However, differences between the two references are minimal $[15,22]$. In a sensitivity analysis we compared our PCD population to $\mathrm{FEV}_{1} \%$ pred values based on GLI reference values published in the 2016 annual data report of the UK CF Registry and found similar results (online supplementary figure S4).

Previous studies examining lung function in patients with PCD included rather small selected patient groups from single centres and have yielded inconsistent results [12]. Our findings suggest strongly that lung function in patients with PCD is already reduced early in life. Different mechanisms could contribute, including reversible causes such as temporary atelectasis and mucus plugging [23], and irreversible damage such as lung remodelling after recurrent severe infections during the period of lung growth [24]. In addition, our data suggest that lung function in adulthood remains impaired. Some recent studies are in line with our findings. A study of 118 adult patients from the UK [11] and a multicentre study including 158 children and adults from the UK, Italy and Denmark [25] both reported reduced 
TABLE 3 Forced expiratory volume in $1 \mathrm{~s}$ (FEV1) and forced vital capacity (FVC) of primary ciliary dyskinesia (PCD) patients of the international PCD Cohort with available ultrastructural defect information compared to Global Lung Function Initiative 2012 reference values

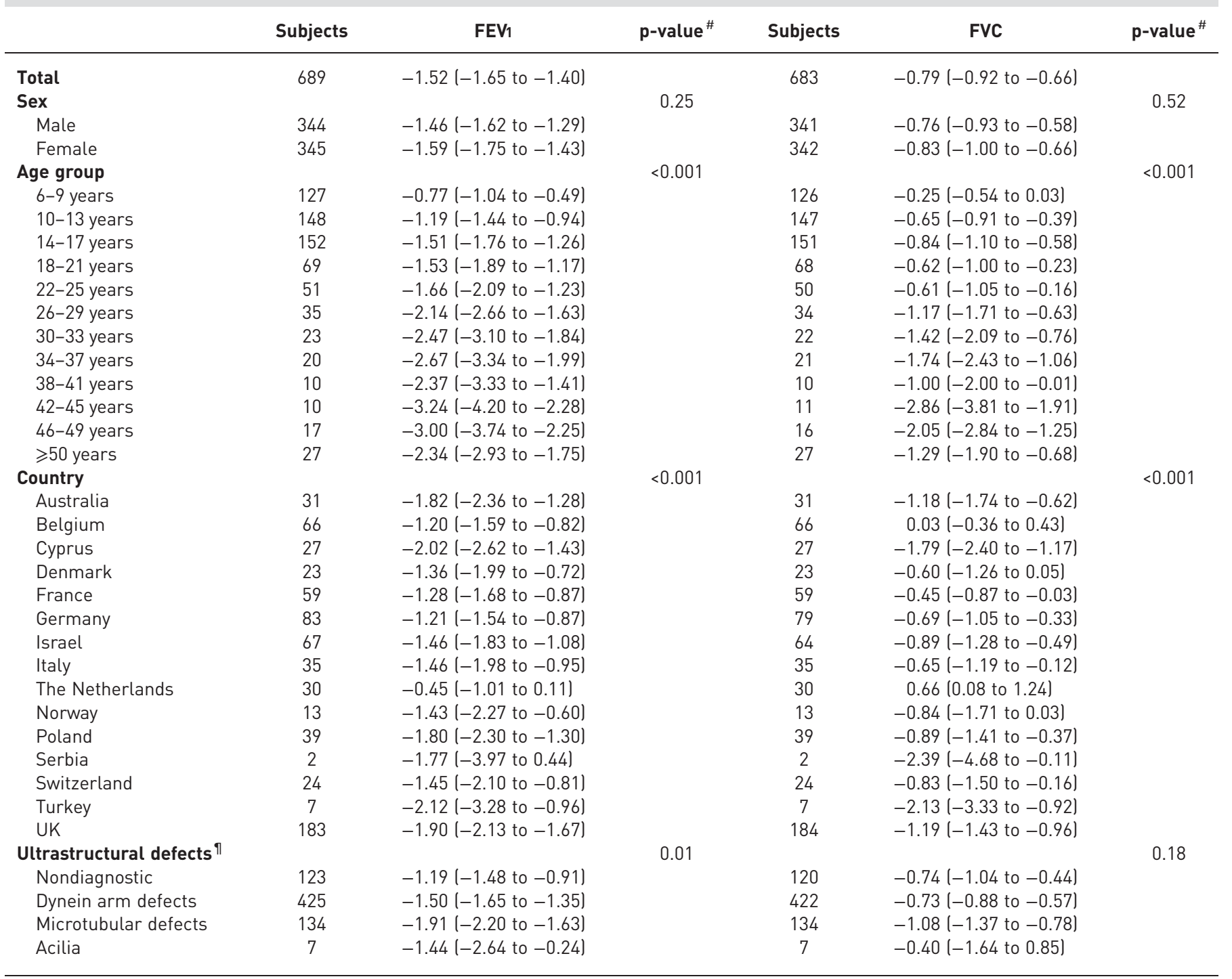

Data are presented as $\mathrm{n}$ or mean z-scores $(95 \% \mathrm{Cl})$ for each group after adjusting for the remaining characteristics, unless otherwise stated. \#: likelihood ratio test $p$-value indicating whether the characteristic explains differences in FEV 1 or FVC within the study population; " ${ }^{\text {\# }}$ dynein arm defects: outer and/or inner dynein arm defects; microtubular defects: central pair, tubulus disorganisation, tubular transposition and/or nexin link defect.

FEV 1 and FVC in patients with PCD. Lung function in 168 patients from Belgium was already reduced at the age of 5 years, but there was no evidence of a difference in FEV1 and FVC $z$-scores between age groups [8]. The wide variation we found between countries could reflect variations in genotype, age at diagnosis [4], management [26] or appropriateness of the GLI reference values for the different countries.

We found that females had a poorer lung function than males (FEV1 Z-scores females -1.57, males -1.36). Although other studies have reported similar sex differences in PCD [9, 27] and CF [28], the processes that affect lung function particularly in females are not well understood. It could reflect treatment variation, differences in therapy adherence or anatomical, hormonal or cultural causes. The female sex hormone progesterone is known to inhibit the function of the mucociliary apparatus and could contribute to the sex disparity [29].

In addition, we found a strong association between lung function and BMI, thus confirming previous results from an overlapping dataset [30]. Data on BMI and lung function in PCD patients from other 

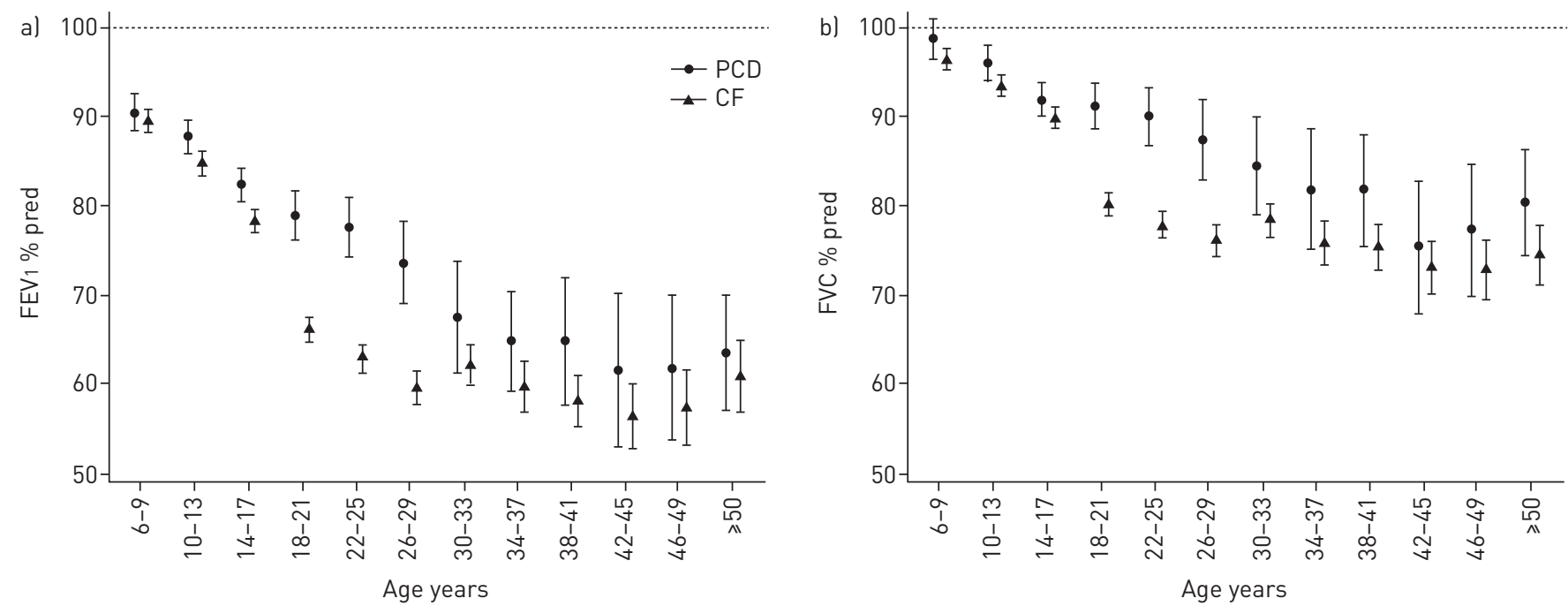

FIGURE 4 Association of al forced expiratory volume in $1 \mathrm{~s}$ (FEV 1 ) and b) forced vital capacity (FVC) of primary ciliary dyskinesia (PCD) patients with cystic fibrosis (CF) patients. FEV1 and FVC are presented as mean \% predicted ( $95 \% \mathrm{CI}$ ), without adjusting for other factors. The dashed line shows the mean of the normal population.

studies are inconsistent. The multicentre study of 158 children found no correlation between BMI and FEV1 z-scores [25], while a study of 34 patients from Israel reported a borderline correlation between FEV1 and BMI in patients with PCD [31]. It has been shown repeatedly that nutritional input can influence outcomes in chronic respiratory diseases [32]. Reduced BMI could be the result of increased energy spent because of an increased respiratory effort, or nutritional intake could lead to both lower BMI and poor lung function [33]. Lastly, purulent lung disease can negatively affect appetite and nutritional status [34]. Information on the direction of association, i.e. whether BMI influences lung function or lung function influences BMI can only be taken from longitudinal studies with repeated measurements of both parameters. However, as intensive nutritional input from an early age has contributed to reduced mortality in CF patients in recent years $[35,36]$ it should also be considered in PCD treatment regimens.

We found that patients with microtubular ultrastructural defects had a lower FEV1 compared to the other patients. Our findings are in line with other recent studies, where patients with microtubular defects had worse lung function measured with spirometry or breath washout techniques than patients with outer and inner dynein arm defects $[7,11,37]$. The mechanisms leading to differences in lung function based on the underlining ultrastructural defect are not understood. However, the findings show a possible link between the genotype and ultrastructural phenotype with the clinical manifestations, possibly due to differences in mechanics of impaired cilia movement. Adequate diagnostic evaluation could provide important information to understand severity and prognosis in PCD.

Studies have reported contradictory results about associations between age at diagnosis and lung function of PCD patients. Some studies reported that regular treatment can maintain lung function [25, 38, 39], and early diagnosis may prevent progression of lung disease [27]. However, other studies reported no association between age at diagnosis and decline in lung function $[10,11]$. We did not see evidence that early diagnosis is associated with better lung function in adolescents. A possible explanation, other than the relatively small subgroup sample could be that patients with milder disease and thus with better lung function are diagnosed later in life than patients with more severe disease. Another possibility is that our studied differences in age of diagnosis might not have been large enough to have a measurable effect on spirometry. Longitudinal studies with more sensitive outcome measures will be necessary to shed further light on the importance of early diagnosis and disease management in patients with PCD.

Comparisons of lung function between PCD and CF are rare and the results have been unclear. Some studies concluded that FEV1 was similar in PCD and CF patients [31, 40, 41], while another reported that it was lower in PCD children compared to pancreatic-sufficient and -insufficient CF children [42]. Yet another study found that children with PCD had better FVC, but similar FEV1 to children with CF [43]. However, all these studies had low numbers and might not be representative of the average patient with PCD. Our findings from a large multinational dataset suggest that lung function in PCD patients is comparable to that of CF patients in childhood, but better in young adulthood. This may suggest that patients with CF have a worse disease course than patients with PCD because they have a more aggressive lung disease. Many of the younger CF patients might have been diagnosed via newborn screening, before 
they developed respiratory symptoms, while older CF patients have been diagnosed later, after initial lung damage has occurred. Patients with PCD in our study have all been diagnosed late, sometimes years or decades after the presentation of symptoms $[4,11]$. This could explain the similar lung function for CF and PCD patients early in life despite a more severe disease course in CF. Additionally, it could be that children with PCD are treated less effectively early in life than those with CF due to the lack of evidence-based treatment recommendations or patients already diagnosed with PCD in childhood have a worse disease course and thus lower lung function than patients diagnosed later in life. The decline of lung function by age was steeper in young adults with $\mathrm{CF}$, and seemed to plateau in middle-aged patients in both diseases. This stabilisation of impaired lung function in middle-aged and older patients might be an effect of mortality or lung transplantation; the surviving patients could be those with a less severe disease course. Unfortunately, there are few data on mortality in patients with PCD [24]. Longitudinal data would allow the evaluation of changes in lung function over time and the identification of predictors of poor lung function later in life. This could assist finding strategies to delay lung function impairment and disease progression.

In conclusion, although PCD has been considered a relatively mild disease, this study suggests that it affects lung function from an early age, similarly to CF. This and the significant variation in PCD across countries emphasise the importance of early, multidisciplinary, standardised care and evidence-based treatments for all patients with PCD.

Acknowledgements: We want to thank all the patients in the iPCD Cohort and their families, and we are grateful to the PCD patient organisations that collaborated closely with us. We thank all the researchers in the participating centres who helped collect and enter data, and worked closely with us throughout the build-up of the iPCD Cohort. We thank Zorica Zivkovic (Children's Hospital for Lung Diseases and TB, Medical Centre "Dr Dragisa Misovic", Belgrade, Serbia) for contributing patients from her centre. We thank Christopher Ritter (Institute of Social and Preventive Medicine, University of Bern, Switzerland) for his editorial suggestions.

Author contributions: C.E. Kuehni, F.S. Halbeisen, M. Goutaki and P. Latzin developed the concept and designed the study. F.S. Halbeisen and M. Goutaki cleaned and standardised the data. F.S. Halbeisen performed the statistical analyses. All other authors participated in discussions for the development of the study and contributed data. C.E. Kuehni, F.S. Halbeisen and M. Goutaki drafted the manuscript. All authors contributed to iterations and approved the final version. C.E. Kuehni and F.S. Halbeisen take final responsibility for the contents.

Conflict of interest: N. Schwerk reports personal fees for lecturing from Novartis, Allergopharma and Infectopharm, and grants from FP7-ChILD EU, outside the submitted work. P. Yiallouros reports grants from European Union's Seventh Framework Programme under EG-GA (number 35404 BESTCILIA), during the conduct of the study. P. Latzin reports personal fees from Gilead, Novartis, Polyphor, Roche, Santhera, Schwabe, Vertex, Vifor and Zambon, outside the submitted work. H. Mazurek reports grants from Bestcilia, during the conduct of the study.

Support statement: This study is supported by Swiss National Science Foundation (320030_173044). The development of the iPCD Cohort has been funded from the European Union's Seventh Framework Programme under EG-GA No.35404 BESTCILIA: Better Experimental Screening and Treatment for Primary Ciliary Dyskinesia. PCD research at ISPM Bern also receives national funding from the Lung Leagues of Bern, St. Gallen, Vaud, Ticino, and Valais, and the Milena-Carvajal Pro Kartagener Foundation. The researchers participate in the network of COST Action BEAT-PCD: Better Evidence to Advance Therapeutic options for PCD (BM 1407). B.D. Spycher was supported by a Swiss National Science Foundation fellowship (PZ00P3_147987). The National PCD Centre in Southampton is commissioned and funded by NHS England. Research in Southampton is supported by NIHR Southampton Biomedical Research Centre, NIHR Wellcome Trust Clinical Research Facility, National Institute for Health Research (RfPB PB-PG-1215-20014) and The AAIR Charity (Reg. No. 1129698). Funding information for this article has been deposited with the Crossref Funder Registry.

\section{References}

1 Barbato A, Frischer T, Kuehni CE, et al. Primary ciliary dyskinesia: a consensus statement on diagnostic and treatment approaches in children. Eur Respir J 2009; 34: 1264-1276.

2 Goutaki M, Meier AB, Halbeisen FS, et al. Clinical manifestations in primary ciliary dyskinesia: systematic review and meta-analysis. Eur Respir J 2016; 48: 1081-1095.

3 Behan L, Dunn Galvin A, Rubbo B, et al. Diagnosing primary ciliary dyskinesia: an international patient perspective. Eur Respir J 2016; 48: 1096-1107.

4 Kuehni CE, Frischer T, Strippoli MP, et al. Factors influencing age at diagnosis of primary ciliary dyskinesia in European children. Eur Respir J 2010; 36: 1248-1258.

5 Lucas JS, Walker WT, Kuehni CE, et al. Primary ciliary dyskinesia. In: Courdier J-F, ed. Orphan Lung Diseases (ERS Monograph). Sheffield, European Respiratory Society, 2011; pp. 201-217.

6 Dell SD. Primary ciliary dyskinesia: myths and realities. Paediatr Child Health 2008; 13: 668-670.

7 Davis SD, Ferkol TW, Rosenfeld M, et al. Clinical features of childhood primary ciliary dyskinesia by genotype and ultrastructural phenotype. Am J Respir Crit Care Med 2015; 191: 316-324.

8 Boon M, Smits A, Cuppens H, et al. Primary ciliary dyskinesia: critical evaluation of clinical symptoms and diagnosis in patients with normal and abnormal ultrastructure. Orphanet J Rare Dis 2014; 9: 11.

9 Frija-Masson J, Bassinet L, Honoré I, et al. Clinical characteristics, functional respiratory decline and follow-up in adult patients with primary ciliary dyskinesia. Thorax 2017; 72: 154-160. 
10 Marthin JK, Petersen N, Skovgaard LT, et al. Lung function in patients with primary ciliary dyskinesia: a cross-sectional and 3-decade longitudinal study. Am J Respir Crit Care Med 2010; 181: 1262-1268.

11 Shah A, Shoemark A, MacNeill SJ, et al. A longitudinal study characterising a large adult primary ciliary dyskinesia population. Eur Respir J 2016; 48: 441-450.

12 Goutaki M, Jose A, Meier B, et al. Lung function of patients with primary ciliary dyskinesia: a systematic review. Eur Respir J 2015; 46: A4518.

13 Goutaki M, Maurer E, Halbeisen FS, et al. The international primary ciliary dyskinesia cohort (iPCD Cohort): methods and first results. Eur Respir J 2017; 49: 1601181.

14 Quanjer PH, Stanojevic S, Cole TJ, et al. Multi-ethnic reference values for spirometry for the 3-95-yr age range: the global lung function 2012 equations. Eur Respir J 2012; 40: 1324-1343.

15 Goss $\mathrm{CH}$, MacNeill SJ, Quinton $\mathrm{HB}$, et al. Children and young adults with CF in the USA have better lung function compared with the UK. Thorax 2015; 70: 229-236.

16 Rubbo B, Behan L, Dehlink E, et al. Proceedings of the COST action BM1407 inaugural conference BEAT-PCD: translational research in primary ciliary dyskinesia - bench, bedside, and population perspectives. BMC Proc 2016; 10: 66 .

17 Lucas JS, Paff T, Goggin P, et al. Diagnostic methods in primary ciliary dyskinesia. Paediatr Respir Rev 2016; 18: 8-17.

18 Lucas JS, Barbato A, Collins SA, et al. European Respiratory Society guidelines for the diagnosis of primary ciliary dyskinesia. Eur Respir J 2017; 49: 1601090.

19 Strippoli MP, Frischer T, Barbato A, et al. Management of primary ciliary dyskinesia in European children: recommendations and clinical practice. Eur Respir J 2012; 39: 1482-1491.

20 Davis MJ. Contrast coding in multiple regression analysis: strengths, weaknesses, and utility of popular coding structures. J Data Sci 2010; 8: 61-73.

21 Wendorf CA. Primer on multiple regression coding: common forms and the additional case of repeated contrasts. Understanding Statistics 2004; 3: 47-57.

22 Stanojevic S, Stocks J, Bountziouka V, et al. The impact of switching to the new global lung function initiative equations on spirometry results in the UK CF registry. J Cyst Fibros 2014; 13: 319-327.

23 Nyilas S, Bauman G, Sommer G, et al. Novel magnetic resonance technique for functional imaging of cystic fibrosis lung disease. Eur Respir J 2017; 50: 1701464.

24 Stocks J, Sonnappa S. Early life influences on the development of chronic obstructive pulmonary disease. Ther Adv Respir Dis 2013; 7: 161-173.

25 Maglione M, Bush A, Nielsen KG, et al. Multicenter analysis of body mass index, lung function, and sputum microbiology in primary ciliary dyskinesia. Pediatr Pulmonol 2014; 49: 1243-1250.

26 Strippoli MP, Frischer T, Barbato A, et al. Management of primary ciliary dyskinesia in European children: recommendations and clinical practice. Eur Respir J 2012; 39: 1482-1491.

27 Yiallouros PK, Kouis P, Middleton N, et al. Clinical features of primary ciliary dyskinesia in Cyprus with emphasis on lobectomized patients. Respir Med 2015; 109: 347-356.

28 Rosenfeld M, VanDevanter DR, Ren CL, et al. Decline in lung function does not predict future decline in lung function in cystic fibrosis patients. Pediatr Pulmonol 2015; 50: 856-862.

29 Jain R, Ray JM, Pan JH, et al. Sex hormone-dependent regulation of cilia beat frequency in airway epithelium. Am J Respir Cell Mol Biol 2012; 46: 446-453.

30 Goutaki M, Halbeisen FS, Spycher BD, et al. Growth and nutritional status, and their association with lung function: a study from the international Primary Ciliary Dyskinesia Cohort. Eur Respir J 2017; 50: 1701659.

31 Cohen-Cymberknoh M, Simanovsky N, Hiller N, et al. Differences in disease expression between primary ciliary dyskinesia and cystic fibrosis with and without pancreatic insufficiency. Chest 2014; 145: 738-744.

32 Liou TG, Adler FR, Fitzsimmons SC, et al. Predictive 5-year survivorship model of cystic fibrosis. Am J Epidemiol 2001; 153: 345-352.

33 Korten I, Usemann J, Latzin P. "Lung sparing growth": is the lung not affected by malnutrition? Eur Respir J 2017; 49: 1700295.

34 Stephensen CB. Burden of infection on growth failure. J Nutr 1999; 129: 534s-538s.

35 Dodge JA, Lewis PA, Stanton M, et al. Cystic fibrosis mortality and survival in the UK: 1947-2003. Eur Respir J 2007; 29: 522-526.

36 Stephenson AL, Mannik LA, Walsh S, et al. Longitudinal trends in nutritional status and the relation between lung function and BMI in cystic fibrosis: a population-based cohort study. Am J Clin Nutr 2013; 97: 872-877.

37 Irving S, Dixon M, Fassad MR, et al. Primary ciliary dyskinesia due to microtubular defects is associated with worse lung clearance index. Lung 2018; 196: 231-238.

38 Ellerman A, Bisgaard H. Longitudinal study of lung function in a cohort of primary ciliary dyskinesia. Eur Respir J 1997; 10: 2376-2379.

39 Hellinckx J, Demedts M, De Boeck K. Primary ciliary dyskinesia: evolution of pulmonary function. Eur J Pediatr 1998; 157: 422-426.

40 Ratjen F, Waters V, Klingel M, et al. Changes in airway inflammation during pulmonary exacerbations in patients with cystic fibrosis and primary ciliary dyskinesia. Eur Respir J 2016; 47: 829-836.

41 Maglione M, Montella S, Mollica C, et al. Lung structure and function similarities between primary ciliary dyskinesia and mild cystic fibrosis: a pilot study. Ital J Pediatr 2017; 43: 34.

42 Walker W, Harris A, Rubbo B, et al. Lung function and nutritional status in children with cystic fibrosis and primary ciliary dyskinesia. Eur Respir J 2016; 48: Suppl. 60, PA3128.

43 Thorpe A, Saint G, Ross A, et al. 336 children with cystic fibrosis have better lung function but comparable growth with age- and sex-matched peers with PCD. J Cyst Fibros 2011; 10: S86. 